\title{
Sufficient Conditions for Output Reference Tracking for Nonlinear Systems: a Contractive Approach
}

\author{
Mattia Giaccagli ${ }^{1}$, Daniele Astolfi ${ }^{1}$, Vincent Andrieu ${ }^{1}$ and Lorenzo Marconi ${ }^{2}$
}

\begin{abstract}
This paper deals with a tracking problem for nonlinear systems. We present sufficient conditions for the state-feedback output tracking problem, in case of arbitrarily large constant references and arbitrarily large domain of attraction. We present an extension of forwarding-based control techniques applied in an incremental framework. Simulations of an academic example are presented to validate the results.
\end{abstract}

\section{INTRODUCTION}

This work deals with the design of control laws for nonlinear systems to track a constant reference set-point. For linear systems this problem has been completely solved in the context of the so-called internal model principle (see [15]) stating that the regulator needs to include an integral action processing the regulated error. Based on this principle, many solutions have been proposed in case of nonlinear systems. We divide these solutions in two main classes.

The first one consists in employing a change of coordinate to put the system into the so-called normal form, [18, Chapter 4.1]. Depending on the properties of the zero-dynamics, different control design have been proposed: see, among others, [9], [20], [22], [26] for minimum-phase systems and [17] for non-minimum phase. In these settings, output tracking can be ensured for "large references" with a semi-global (or global) domain of attraction. However, such designs cannot be applied when the relative degree between the regulated output and the control is not well-defined, namely when the normal form doesn't exist. Furthermore, the extension to multi-input multi-output (and possibly more input than regulated output) is not trivial. See for instance, [11], [34].

The second class of approaches follows the linear paradigm: 1) extend the controlled system with an integral action processing the regulated errors; 2) stabilize the unperturbed (i.e. with zero-reference) extended system; 3) finally analyze the behaviour of the closed-loop system in presence of the reference. Such approach is in general more flexible as it's "coordinate-free" and can be easily extended to multiinput multi-output cases, but it's limited in the size of the domain of attraction and the reference, see for instance [23, Chapter 12.4] with a linearization-based feedback design. In order to relax such constraints, forwarding design (see, for instance, [21], [27], for a review) have been proposed in [6], [30] so that to achieve semi-global domain of attractions.

\footnotetext{
${ }^{1}$ Univ Lyon, Université Claude Bernard Lyon 1, CNRS, LAGEPP UMR 5007, 43 boulevard du 11 novembre 1918, F-69100, Villeurbanne, France.

${ }^{2}$ CASY - DEI, University of Bologna, Italy.

Authors acknowledge Université de Lyon 1 and Ecole Doctorale EEA for funding the $\mathrm{PhD}$ thesis of the first author.
}

Nevertheless, the size of the reference still need to be "small".

The objective of this work is therefore to study sufficient conditions to design a state feedback control law for the problem of output set-point tracking for nonlinear systems with a global domain of attraction and without restrictions on the amplitude of the set-point. We aim at proposing conditions that are "coordinate-free", i.e. we don't look for any normal form. For this, we follow similar ideas to [29] by casting the output tracking problem into the contraction theory and we propose a new control design based on a modification of forwarding-based techniques able to preserve the desired incremental stability properties, see, for instance, [2], [5], [14], [25] and references therein. While the extension to the incremental framework has already been done for several control techniques, such as backstepping [36] [37] [32], LMI [13] and circle criterion [35] [33], to the best of authors knowledge the problem of incremental forwarding hasn't been addressed in literature. Yet, forwarding is the natural tool to stabilize systems in cascade form as the one obtained when the regulated system is extended with integral actions, see [6], [30]. First we present sufficient conditions for the incremental stabilization problem via forwarding. In this context we state a non-uniform global incremental exponential stability result, which can be made uniform under more restrictive conditions. Successively, we apply the proposed design to the output set-point reference tracking problem. We establish sufficient conditions to design a stabilizing feedback law to solve such a problem for arbitrarily large references and for arbitrarily large domain of attraction. The solution is coordinate-free and does not require the knowledge of the set point in the design of the controller.

The paper is structured as follows. First, in Section II, we present the problem statement and we provide some preliminaries results in order to clarify the link between contraction theory and output tracking. Then, in Section III, we recall some results on forwarding design and we give sufficient conditions and a constructive design to enforce incremental stability properties. Finally, in Section IV, we give the main results on output tracking. An academic example is given in Section V. Conclusions are derived in Section VI. Parts of the proofs are postponed to the Appendix.

Notation: We define $\mathbb{R}_{>0}=(0, \infty)$ and $\mathbb{R}_{\geq 0}=[0, \infty)$. Given a set $\mathcal{A} \subset \mathbb{R}^{n}$, we denote with $\operatorname{Cl}(\mathcal{A})$ its closure. Given $x \in \mathbb{R}^{n}, y \in \mathbb{R}^{m}$, we denote $(x, y)=\left(x^{\top}, y^{\top}\right)^{\top}$. Given a matrix $A$ we denote with $|A|$ the standard induced matrix norm. We denote with $I_{n}$ the Identity matrix of 
dimension $n \times n$; when there's no possibility of misunderstanding we drop the index and use simply $I$. Given two vector fields $f: \mathbb{R}^{n} \rightarrow \mathbb{R}^{n}, h: \mathbb{R}^{n} \rightarrow \mathbb{R}$, we denote the Lie derivative of $h$ along $f$ as $L_{f} h(x):=\frac{\partial h}{\partial x}(x) f(x)$.

\section{PReliminaries}

\section{A. Problem Statement}

In this paper we are interested to the output set-point tracking problem for nonlinear systems of the form

$$
\dot{x}=f(x)+g(x) u, \quad y=h(x),
$$

where $x \in \mathbb{R}^{n}$ is the system state, $u \in \mathbb{R}$ is the control input and $y \in \mathbb{R}$ is the output. The goal is to design a state feedback control law $u$ such that, given a reference $r \in \mathbb{R}$, the closed loop system trajectories are bounded and, asymptotically, $\lim _{t \rightarrow \infty} e(t):=h(x(t))-r=0$.

In this paper we are interested in a coordinate-free approach, namely, we look for a solution that doesn't involve the use of normal forms of (1) by working on the "original coordinates" restriction on the relative degree of the system.

In the spirit of [6], [30], we extend system (1) with an integral action

$$
\dot{\eta}=h(x)-r,
$$

and we look for a state-feedback law of the form

$$
u=\psi(\eta, x),
$$

which leads to a closed loop system

$$
\dot{\mathcal{X}}=\varphi(\mathcal{X})+\Gamma r
$$

where $\mathcal{X}=(x, \eta)$ and

$$
\varphi(\mathcal{X})=\left[\begin{array}{c}
f(x)+g(x) \psi(\eta, x) \\
h(x)
\end{array}\right], \quad \Gamma=\left[\begin{array}{c}
0 \\
-1
\end{array}\right] .
$$

This type of control law solves the tracking problem if an equilibrium $\mathcal{X}^{*}=\left(x^{\star}, \eta^{\star}\right)$ exists, is unique and is globally asymptotically stable. As a matter of fact, in this case, $h\left(x^{\star}\right)-r=0$ and consequently the error $e$ converges to zero along any solution of the closed loop system.

Constructing the feedback $\psi$ such that, for all $r$, an equilibrium exists and is globally asymptotically stable is not an easy task when the system is not in normal form. The results presented in [6], [30] provide a solution that however poses limitations on the domain of attraction and on the amplitude of the references, in the sense that they provide a solution that is semi-global in $\mathcal{X}$ and only local in the size of $r$. To overcome this constraints, we take advantage of contraction theory, which is shortly recalled in the next section.

\section{B. Incremental Stability and Contraction Theory}

Consider a systems of the form

$$
\dot{\mathcal{X}}=\varphi(\mathcal{X})
$$

where $\mathcal{X} \in \mathbb{R}^{\varrho}$ is the state and $\varphi$ is a $C^{1}$ vector field. By $\mathcal{X}\left(\mathcal{X}_{0}, t\right)$ we denote the trajectory of the system starting from the initial condition $\mathcal{X}_{0}$ at time $t=0$.
In the following we will also adopt the following notation. Given a 2 tensor $\mathcal{P}: \mathbb{R}^{\varrho} \rightarrow \mathbb{R}^{\varrho \times \varrho}$ and the vector field $\varphi$ : $\mathbb{R}^{\varrho} \rightarrow \mathbb{R}^{\varrho}$, we denote the Lie derivative of the tensor $\mathcal{P}$ along $\varphi$ as $L_{\varphi} \mathcal{P}(\mathcal{X})$, defined as

$$
L_{\varphi} \mathcal{P}(\mathcal{X}):=\mathfrak{d}_{\varphi} \mathcal{P}(\mathcal{X})+\left(\mathcal{P}(\mathcal{X}) \frac{\partial \varphi}{\partial \mathcal{X}}(\mathcal{X})\right)+\left(\mathcal{P}(\mathcal{X}) \frac{\partial \varphi}{\partial \mathcal{X}}(\mathcal{X})\right)^{\top}
$$

where

$$
\mathfrak{d}_{\varphi} \mathcal{P}(\mathcal{X}):=\lim _{h \rightarrow 0} \frac{\mathcal{P}(\mathcal{X}(\mathcal{X}, h))-\mathcal{P}(\mathcal{X})}{h} .
$$

Definition 1. We say that system (6) is Incrementally Asymptotically Stable (in short IAS) on a positively invariant subset $\mathcal{O}$ of $\mathbb{R}^{\varrho}$ if there exists a class $\mathcal{K} \mathcal{L}$ function $\alpha$ such that

$$
\left|\mathcal{X}\left(\mathcal{X}_{1}, t\right)-\mathcal{X}\left(\mathcal{X}_{2}, t\right)\right| \leq \alpha\left(\left|\mathcal{X}_{1}-\mathcal{X}_{2}\right|, t\right),
$$

for any $\mathcal{X}_{1}, \mathcal{X}_{2} \in \mathcal{O}$, and for any $t \geq 0$. If $\mathcal{O}=\mathbb{R}^{\varrho}$, then we say that system (6) is Incrementally Globally Asymptotically Stable (in short IGAS).

IGAS property states that if we pick two different initial conditions of system (6), the two trajectories that evolve in time starting from these initial conditions, will converge with each other as time goes on. Incremental properties are useful, for instance, whenever we are not interested in the convergence of trajectories toward an equilibrium point, but rather to study the relative behaviors of trajectories. In case the function $\alpha$ in (7) has an exponential decay, we have the following definition.

Definition 2. We say that system (6) is Incrementally Exponentially Stable (in short IES) on a positively invariant subset $\mathcal{O}$ of $\mathbb{R}^{\varrho}$ if there exist $k, \lambda \in \mathbb{R}_{>0}$ such that

$$
\left|\mathcal{X}\left(\mathcal{X}_{1}, t\right)-\mathcal{X}\left(\mathcal{X}_{2}, t\right)\right| \leq k\left|\mathcal{X}_{1}-\mathcal{X}_{2}\right| \exp (-\lambda t)
$$

for all $\mathcal{X}_{1}, \mathcal{X}_{2} \in \mathcal{O}, t \geq 0$. If $\mathcal{O}=\mathbb{R}^{\varrho}$, then we say that (6) is Incrementally Globally Exponentially Stable (in short IGES).

It is well known that IGAS/IGES properties are related to differential properties on the vector field $\varphi$. We recall below an important result linking incremental stability properties and the existence of a metric which decreases along the vector field.

Theorem 1. If there exists a $C^{1}$ function $\mathcal{P}: \mathbb{R}^{\varrho} \rightarrow \mathbb{R}^{\varrho \times \varrho}$ taking positive definite symmetric values, and positive real numbers $\overline{\mathfrak{p}}, \underline{\mathfrak{p}}, \mathfrak{q} \in \mathbb{R}_{>0}$ such that

$$
\begin{aligned}
\underline{\mathfrak{p}} I_{\varrho} \leq \mathcal{P}(\mathcal{X}) & \leq \overline{\mathfrak{p}} I_{\varrho} \\
L_{\varphi} \mathcal{P}(\mathcal{X}) & \leq-\mathfrak{q} I_{\varrho}
\end{aligned}
$$

for all $\mathcal{X} \in \mathbb{R}^{\varrho}$, then system (6) is IGES. Conversely, if system (6) is IGES and $\varphi$ is $C^{2}$ with bounded first and second derivatives, then there exist a $C^{1}$ function $\mathcal{P}$ and $\underline{\mathfrak{p}}, \overline{\mathfrak{p}}, \mathfrak{q} \in \mathbb{R}_{>0}$ satisfying (9), (10).

Proof. The proof of the first part of the theorem can be found in [24, Theorem 1], [2, Section II-B], [14]. The converse can be found for instance in [2, Proposition 4]. 
When inequality (10) holds on the (Riemannian) convex hull of a positively invariant compact set, exponential contraction is established in this one as stated in the following proposition.

Proposition 1. Assume that there exists a $C^{1}$ function $\mathcal{P}$ : $\mathbb{R}^{\varrho} \rightarrow \mathbb{R}^{\varrho \times \varrho}$ taking positive definite symmetric values, and positive real numbers $\overline{\mathfrak{p}}, \mathfrak{p}$, such that (9) holds for all $\mathcal{X}$ in $\mathbb{R}^{\varrho}$. Let $\mathcal{C}$ be compact invariant subset of $\mathbb{R}^{\varrho}$ for the dynamical system (6) and assume that there exists $\mathfrak{q} \in \mathbb{R}_{>0}$ such that (10) holds for all $\mathcal{X}$ in $\mathcal{O}$ defined as

$$
\mathcal{O}=\left\{\mathcal{X} \in \mathbb{R}^{\varrho}, \max _{w \in \mathcal{C}}|\mathcal{X}-w|<\varpi\right\},
$$

where

$$
\varpi>\frac{\sqrt{\overline{\mathfrak{p}}}}{2 \sqrt{\underline{\mathfrak{p}}}} \max _{\left(\mathcal{X}_{a}, \mathcal{X}_{b}\right) \in \mathcal{C}^{2}}\left|\mathcal{X}_{a}-\mathcal{X}_{b}\right|,
$$

then system (6) is IES on $\mathcal{C}$.

Proof. The proof is omitted for space reasons. It can be deduced by carefully specializing similar arguments to those employed in Theorem 1.

Finally, we recall below the notion of "Killing Vector Field" (see, for instance, [12, Appendix B]).

Definition 3. Given $C^{1}$ functions $\mathcal{P}: \mathbb{R}^{\varrho} \mapsto \mathbb{R}^{\varrho \times \varrho}$, and $\gamma: \mathbb{R}^{\varrho} \rightarrow \mathbb{R}^{\varrho}$, we say that $\gamma$ is a Killing Vector Field for $\mathcal{P}$ if $L_{\gamma} \mathcal{P}(\mathcal{X})=0$ for all $\mathcal{X} \in \mathbb{R}^{\varrho}$.

\section{From contraction to regulation}

Our motivation to study incremental stability property comes from the following result, stating that the tracking problem for system (1) is automatically solved if we are able to make the closed-loop system (4) IGES.

Theorem 2. Assume there exists a $C^{1}$ function $\psi: \mathbb{R} \times \mathbb{R}^{n} \mapsto$ $\mathbb{R}$ such that for all $r$ in $\mathbb{R}$ the control law (3) ensures that the closed loop system (4) is IGES. Then, this control law is a solution to the (global) output tracking problem stated in Section II-A i.e. $\lim _{t \rightarrow \infty} y(t)=r$ for any initial condition $\mathcal{X}_{0}=\left(\eta_{0}, x_{0}\right)$ in $\mathbb{R}^{n+1}$.

Instrumental to the proof of Theorem 2, we have the following Lemma.

Lemma 1. Suppose system (6) is IES in a closed forward invariant set $\mathcal{C} \subset \mathbb{R}^{\varrho}$. Then, there exists a unique equilibrium point $\mathcal{X}^{\star} \in \mathcal{C}$ which attracts all solutions initiated from $\mathcal{C}$.

\section{Proof. See Appendix A.}

Remark 1. Interestingly, a straightforward consequence of Lemma 1 is that trajectories of a IES system evolving in a closed forward invariant set cannot converge to a limit cycles, but only to an equilibrium.

Proof of Theorem 2. By applying Lemma 1 with $\mathcal{C}=\mathbb{R}^{\varrho}$, we know the existence of unique equilibrium point denoted $\mathcal{X}^{\star}=\left(x^{\star}, \eta^{\star}\right)$. Note that $\mathcal{X}^{\star}$ being an equilibrium, it implies, by definition of the $\eta$-dynamics in (2), that $h\left(x^{\star}\right)=r$. System (4) being IGES, there exists $k, \lambda \in \mathbb{R}_{>0}$ such that equation (8) holds which implies that, for all initial conditions $\mathcal{X}^{\circ} \in \mathbb{R}^{n}$, we have

$$
\left|\mathcal{X}\left(\mathcal{X}^{\circ}, t\right)-\mathcal{X}^{\star}\right| \leq k\left|\mathcal{X}^{\circ}-\mathcal{X}^{\star}\right| \exp (-\lambda t) .
$$

Hence Theorem 2 holds.

The statement of Theorem 2 is based on the formal definition of IGES given in Definition (2). However, motivated by the sufficient conditions of Theorem 1, an equivalent result can be also stated in the metric framework, that is by asking for the existence of a feedback law $\psi$ and metric $\mathcal{P}$ satisfying the properties (9), (10) for the closed-loop system (4). Moreover, in the particular case in which the vector $\Gamma$ defined in (4) is a Killing Vector Field for $\mathcal{P}$, it follows that

$$
L_{\varphi+r \Gamma} \mathcal{P}(\mathcal{X})=L_{\varphi} \mathcal{P}(\mathcal{X})+r L_{\Gamma} \mathcal{P}(\mathcal{X})=L_{\varphi} \mathcal{P}(\mathcal{X})
$$

for all $r \in \mathbb{R}$, namely the Killing Vector property is invariant with respect to the size of $r$. As a consequence, based on these remarks, we can state the following result.

Corollary 1. Suppose there exist a $C^{1}$ function $\psi: \mathbb{R} \times$ $\mathbb{R}^{n} \mapsto \mathbb{R}$, a $C^{1}$ function $\mathcal{P}: \mathbb{R}^{m} \rightarrow \mathbb{R}^{m \times m}$ taking positive symmetric values and $\overline{\mathfrak{p}}, \mathfrak{p}, \mathfrak{q} \in \mathbb{R}_{>0}$ such that, for the closed-loop system (4), inequalities (9), (10) hold. Assume, in addition, that $\Gamma$ is a Killing Vector Field for $\mathcal{P}$. Then, for all $r \in \mathbb{R}$ the feedback law (3) solves the global tracking problem, i.e. $\lim _{t \rightarrow \infty} y(t)=r$.

Motivated by this analysis, in the following section, we consider the problem of designing a control law $\psi$ ensuring the contraction property along the vector field $\varphi$ for some $\mathcal{P}$ and the Killing Vector Field property with respect to the vector $\Gamma$. Furthermore, note that, in view of its structure, see (5), the vector field $\Gamma$ acts only in the directions of $\eta$. Hence, it suffices to find a metric $\mathcal{P}$ independent of $\eta$ to have the desired Killing Vector property satisfied.

\section{INCREMENTAL FORWARDING}

\section{A. Highlights on Forwarding Design}

Our system (1)-(2) is in the so-called feedforward form (see [27]). The stabilization of this class of systems has been widely studied in the last years through forwarding control techniques (see [30], [6], [21] and the references therein). The objective of this section is to design a forwarding-based control law ensuring the desired contractivity properties. For this, we first recall the following result.

Lemma 2. Consider system (1), (2) and suppose that the origin of $\dot{x}=f(x)$ is globally asymptotically stable and locally exponentially stable. Then, there exists a $C^{1}$ function $M: \mathbb{R}^{n} \rightarrow \mathbb{R}$ solution of

$$
L_{f} M(x)=h(x) \text {. }
$$

Proof. The proof can be found in [27, Lemma IV.2] or [19, Section 5.2].

Based on the function $M$ defined above, we can define a stabilizing state-feedback control law for the extended system (1)-(2) as

$$
u=\ell(x) L_{g} M(x)(\eta-M(x)),
$$


where $\ell: \mathbb{R}^{n} \rightarrow \mathbb{R}_{>0}$ is a sufficiently small function. See, for instance, [30, equation (19)] for further details. We have then the following result.

Lemma 3. Consider system (1), (2) and let the assumptions stated in Lemma 2 hold. Furthermore, suppose the following condition hold

$$
\left|L_{g} M(0)\right|>0 \text {. }
$$

Then, there exists $\ell: \mathbb{R}^{n} \rightarrow \mathbb{R}_{>0}$ such that the origin of system (1), (2) in closed-loop with (14) is globally asymptotically stable and locally exponentially stable for $r=0$.

Proof. The proof can be found in [6, Lemma 1], [30, Assumption 4] and references therein.

Condition (15) is obtained if the extended system (1), (2) is controllable at the origin. Such property, also known as "nonresonance condition" in regulation theory [6, Assumption 3], corresponds to the condition $C A^{-1} B$ is non zero, where

$$
A:=\left.\frac{\partial f}{\partial x}(x)\right|_{x=0}, \quad B:=g(0), \quad C:=\left.\frac{\partial h}{\partial x}(x)\right|_{x=0} .
$$

\section{B. Assumptions}

Our objective is to design a forwarding-based control law ensuring the desired incremental stability properties for the extended system (1), (2). To this end, we first suppose that the system (1), with $u=0$, is IGES, as stated below.

Assumption 1. There exists a $C^{1}$ function $P: \mathbb{R}^{n} \rightarrow \mathbb{R}^{n \times n}$ taking positive symmetric values and positive real numbers $\bar{p}, \underline{p}, q \in \mathbb{R}_{>0}$ such that, for the vector field $f$ of system (1), inequalities (9) and (10) hold for all $x \in \mathbb{R}^{n}$.

Note that Assumption 1 can be also satisfied after a preliminary state-feedback, similarly to what assumed in [6]. and corresponds to a (incremental) stabilizability assumption of system (1), which is, as a matter of fact, necessary in the linear context, see [15]. For instance, it can be obtained by following the design techniques in [29, Chapter 5], or [3], [4], [28], [36].

Due to Lemma 1, it follows that $\dot{x}=f(x)$ admits the existence of an equilibrium which we assume to be the origin (without loss of generality). Moreover, such equilibrium is globally asymptotically stable and locally exponentially stable and therefore, we can apply Lemma 2 to establish the existence of a function $M$ solution to (13). Similarly to the condition (15) stated in Lemma 3, we need a controllabilitylike condition for $L_{g} M(x)$ which, in our incremental framework, is stated as follows.

Assumption 2. Let $M$ be solution to (13). There exists $m \in$ $\mathbb{R}_{>0}$ such that $\left|L_{g} M(x)\right| \geq m$ for all $x \in \mathbb{R}^{n}$.

Assumption 2 can be read as a uniform controllability-like assumption for extended dynamics (1), (2) and represents the "incremental version" of condition (15) stated in Lemma 3. In particular, the new condition is assumed to hold for all $x$ and not just for the linearized system around the origin as for (15). Finally, we have the following assumption.
Assumption 3. Let $P$ be given by Assumption 1. Then the vector field $g$ of system (1) is a Killing Vector Field for $P$, i.e., $L_{g} P(x)=0$ for all $x \in \mathbb{R}^{n}$.

Assumption 3 guarantees that the (Riemaniann) metric induced by $P$ is invariant along $g$. This means that the system preserves its contractive properties in the directions provided by $g$, namely, in the directions in which the control law acts. Notice that if $g$ is constant, Assumption 3 is satisfied for every constant metric $P$.

\section{Non-uniform Incremental Stability}

In order to solve the problem, taking inspiration from the control law defined in (14), we focus on feedback laws of the form

$$
u=\psi(\eta, x):=\kappa L_{g} M(x)^{-1} \beta(\eta-M(x))
$$

where $M$ is defined in (13), $\kappa \in \mathbb{R}_{>0}$ and $\beta$ is a $C^{1}$ increasing function. We have the following result.

Theorem 3. Let Assumptions 1, 2 and 3 hold. Assume that the function $M$ satisfying (13) is $C^{2}$, and that there exist positive real numbers $\bar{g}, k_{0}, k_{1} \in \mathbb{R}_{>0}$ satisfying

$$
|g(x)| \leq \bar{g},\left|\frac{\partial M}{\partial x}(x)\right| \leq k_{0},\left|\frac{\partial L_{g} M}{\partial x}(x)\right| \leq k_{1},
$$

hold for all $x \in \mathbb{R}^{n}$. Finally, let $\beta: \mathbb{R} \rightarrow \mathbb{R}$ be any $C^{1}$ function satisfying ${ }^{1}$

$$
|\beta(s)| \leq \frac{1}{k_{1}}, \quad 0<\beta^{\prime}(s) \leq 1, \quad \forall s \in \mathbb{R} .
$$

Then, there exists a positive real number $\kappa^{\star}>0$ such that, for any $\kappa \in\left(0, \kappa^{\star}\right)$, the closed-loop system (4), (5), with $\psi$ defined as in (16), admits a $C^{1}$ function $\mathcal{P}: \mathbb{R}^{n+1} \rightarrow$ $\mathbb{R}^{(n+1) \times(n+1)}$, a positive continuous function $\mu: \mathbb{R}^{n+1} \rightarrow$ $\mathbb{R}_{>0}$ and positive real number $\overline{\mathfrak{p}}, \underline{\mathfrak{p}} \in \mathbb{R}_{>0}$ satisfying (9) and

$$
L_{\varphi} \mathcal{P}(\mathcal{X}) \leq-\mu(\mathcal{X}) I_{n+1} \quad \forall \mathcal{X} \in \mathbb{R}^{n+1} .
$$

Moreover, $\Gamma$ is a Killing Vector Field for $\mathcal{P}$.

\section{Proof. See Appendix B}

Note that we can't conclude from (19) that the closed loop system is IGES. Indeed, the right hand side of (19) may be not uniform. This would be the case if $\mu(\eta, x)$ were lower bounded. However, from the proof, we see that this is not possible since $\mu$ depends on $\beta$, and, to have $\mu$ bounded from below, $\beta^{\prime}$ should be lower bounded by a positive real number that, in turn, would violate (18). Note however, that if there exists a compact set which is invariant along the solutions of the system, then continuity of $\mu$ implies that the latter can be uniformly bounded from below and uniform incremental stability holds.

\footnotetext{
${ }^{1}$ For instance, one may select $\beta(s)=\frac{1}{2 \pi k_{1}} \arctan \left(2 \pi k_{1} s\right)$.
} 


\section{Global Incremental Stability}

In order to obtain a global contractivity, we need to suppose a stronger version of Assumption 2. In particular, we need, not only the rank of $L_{g} M$ to be constant, but also the function itself, as stated below.

Assumption 4. There exists a real number $c \neq 0$ such that $L_{g} M(x)=c$ for all $x \in \mathbb{R}^{n}$.

Under previous assumption, we have the following result.

Theorem 4. Let Assumptions 1, 3 and 4 hold. Assume that the function $M$ satisfying (13) is $C^{2}$ and that there exist positive real numbers $\bar{g}, k_{0} \in \mathbb{R}_{>0}$ satisfying

$$
|g(x)| \leq \bar{g}, \quad\left|\frac{\partial M}{\partial x}(x)\right| \leq k_{0} .
$$

for all $x \in \mathbb{R}^{n}$. Then, for any $\kappa \in \mathbb{R}_{>0}$, the closed-loop system (4), (5) with $\psi$ defined as

$$
u=\psi(\eta, x):=\frac{\kappa}{c}[\eta-M(x)]
$$

admits a $C^{1}$ function $\mathcal{P}: \mathbb{R}^{n+1} \rightarrow \mathbb{R}^{(n+1) \times(n+1)}$ taking positive symmetric values and real numbers $\underline{\mathfrak{p}}, \overline{\mathfrak{p}}, \mathfrak{q} \in \mathbb{R}_{>0}$ satisfying (9), (10), for all $\mathcal{X} \in \mathbb{R}^{n+1}$. Moreover, $\Gamma$ is a Killing Vector Field for $\mathcal{P}$.

Proof. See Section C.

\section{INCREMENTAL INTEGRAL ACTION FOR OUTPUT TRACKING}

Finally, by using the feedback design proposed in Section III and the framework presented in Section II, we have the following results on output tracking, based on the design proposed in Theorem 3.

Proposition 2. Suppose all assumptions of Theorem 3 hold and consider system (1) in closed-loop with

$$
u=\psi(\eta, x), \dot{\eta}=h(x)-r,
$$

where $\psi$ is chosen as in Theorem 3. Then, for all constant $r \in \mathbb{R}$ and all $\mathcal{X}_{0}=\left(\eta_{0}, x_{0}\right)$ in $\mathbb{R}^{n+1}$ such that $\mathcal{X}\left(\mathcal{X}_{0}, t\right)$ is bounded in positive time, the output tracking problem is solved, i.e. $\lim _{t \rightarrow \infty} y(t)=r$ for all such initial conditions.

Proof. Applying Theorem 3, picking $\kappa \in\left(0, \kappa^{\star}\right)$, it yields the existence of a $C^{1}$ function $\mathcal{P}: \mathbb{R}^{n+1} \rightarrow \mathbb{R}^{(n+1) \times(n+1)}$, a positive function $\mu: \mathbb{R}^{n+1} \rightarrow \mathbb{R}_{>0}$ such that (19) holds. Let $r$ in $\mathbb{R}$ and $\mathcal{X}_{0}=\left(\eta_{0}, x_{0}\right)$ in $\mathbb{R}^{n+1}$ such that $\mathcal{X}\left(\mathcal{X}_{0}, t\right)$ is bounded in positive time. Let

$$
\mathcal{C}:=\operatorname{Cl}\left\{\bigcup_{t \geq 0}^{\infty} \mathcal{X}\left(\mathcal{X}_{0}, t\right)\right\} .
$$

and note that $\mathcal{C}$ is a forward invariant compact set (by Birkhoff theorem [18, p. 517]). Let $q=\min _{\{(\eta, x) \in O\}} \mu(\eta, x)>0$ where $\mathcal{O}$ is the set defined in (11) for some positive real number $\varpi$ satisfying (12). Keeping in mind that $\Gamma$ is a Killing Vector Field for $\mathcal{P}$, it yields

$$
L_{\varphi+r \Gamma} \mathcal{P}(\mathcal{X})=L_{\varphi} \mathcal{P}(\mathcal{X})+r L_{\Gamma} \mathcal{P}(\mathcal{X}) \leq-q I_{n+1}
$$

for all $\mathcal{X} \in \mathcal{O}$. Hence with Proposition 1, the system is IES in $\mathcal{C}$. With Lemma 1 , it implies the existence of a unique equilibrium $\mathcal{X}^{\star}=\left(\eta^{*}, x^{*}\right) \in \mathcal{C}$ attracting all solution starting from $\mathcal{C}$. Hence, because of the integral action, it yields $h\left(x^{*}\right)=r$.

Note that the boundedness requirement on the trajectory implies most of the time a bound for the reference $r$ that can be tracked. The following result shows that the contractivity property always holds provided that $r$ is sufficiently small. This is a local result in the reference signal but global in the state, (i.e. in the initial conditions). In this respect, the results extends the semi-global result obtained in [6, Proposition 3].

Proposition 3. Suppose all assumptions of Theorem 3 hold. Then there exists $\bar{r} \geq 0$ such that for all $r$ such that $|r| \leq \bar{r}$, the feedback (22) given in Proposition 2 with $\psi$ chosen as in Theorem 3, solves the output tracking problem for system (1), i.e. $\lim _{t \rightarrow \infty} y(t)=r$ for any $|r| \leq \bar{r}$ and for any initial condition $\mathcal{X}_{0}=\left(\eta_{0}, x_{0}\right)$ in $\mathbb{R}^{n+1}$.

Proof. Consider the closed-loop system (4), with $\varphi, \Gamma$ defined as in (5), and $\psi$ selected as in (16). For $r=0$, the origin of (4) is an equilibrium, and because of Theorem 3, it is locally exponentially stable. This can be proved by using the linear approximation at the origin and the Lyapunov function $V(\mathcal{X})=\mathcal{X}^{\top} \mathcal{P}(0) \mathcal{X}$, with $\mathcal{P}(x)$ satisfying (19). Hence, by [6, Lemma 5], there exists $\bar{r}>0$ such that, for all $|r| \leq \bar{r}$, system (4), admits an equilibrium $\mathcal{X}^{*} \in \mathbb{R}^{n+1}$ which is locally exponentially stable, with a domain of attraction $\mathcal{N} \subset \mathbb{R}^{n+1}$. Now, because of Theorem 3, system (4) satisfies (19). In particular, $\Gamma$ is a Killing Vector and $L_{\varphi} \mathcal{P}(\mathcal{X}) \leq 0$ for all $\mathcal{X} \in \mathbb{R}^{n+1}$. This implies that the (Riemannian) distance of any two trajectories of (4) is not increasing, and, in particular, the distance of any trajectory to $\mathcal{X}^{*}$ is not increasing. In other words, the trajectory of (4) is bounded for any initial condition $\mathcal{X}_{0} \in \mathbb{R}^{n+1}$. Hence, the statement of the proof follows by direct application of Proposition 2.

Finally, the last result employs Theorem 4 to obtain a global result.

Proposition 4. Suppose all assumption of Theorem 4 holds. Then the control law (22) with $\psi$ chosen in (21) solves the global output tracking problem for system (1), i.e. $\lim _{t \rightarrow \infty} y(t)=r$ for any $r \in \mathbb{R}$ and for any initial condition $\mathcal{X}_{0}=\left(\eta_{0}, x_{0}\right)$ in $\mathbb{R}^{n+1}$.

Proof. This proposition is a direct consequence of Corollary 1 and Theorem 4.

Note that in [6], [30], the domain of attraction is semiglobal in $\mathcal{X}$ and local (i.e. small) in the references $r$. Differently here, we provide a result that is global (i.e. arbitrarily large) both in term of domain of attraction and reference to be tracked. 


\section{EXAMPLE}

Consider system (1) with

$f(x)=\left(\begin{array}{c}-2 x_{1}+3 x_{2}+\sin \left(x_{1}\right)-x_{1}^{3} \\ -2 x_{2}\end{array}\right), \quad g(x)=\left(\begin{array}{c}1 \\ -1\end{array}\right)$,

and $h(x)=x_{1}^{3}-\sin \left(x_{1}\right)+x_{2}$. First, we can verify that this system verifies Assumption 1 with $P=\operatorname{diag}\left(1, \frac{1}{2}\right)$, $q=\frac{1}{2}, \underline{p}=\frac{1}{2}, \bar{p}=1$. Furthermore, the function $M(x)$ defined in Lemma 2 can be computed as $M(x)=-x_{1}-$ $2 x_{2}$. Hence, Assumptions 2 and 4 are also verified since $L_{g} M(x)=1$. Finally, Assumption 3 is trivially satisfied since $P$ and $g$ are constant. Note also that the relative degree ${ }^{2}$ is not well defined, since $L_{g} h(x)=3 x_{1}^{2}-\cos \left(x_{1}\right)+1$ is not constant. Hence, techniques based on normal forms for output tracking (see for instance [22]) doesn't apply in this case. Nevertheless, we can apply results of Theorem 4 and Proposition 4 to achieve global output tracking. Figures 1 and 2 show the state $x$ and the output $y$ of the system in closed-loop with the control law (21), with $\kappa=10$, when $r=0$ and for different initial conditions selected as $\mathcal{X}(0)=$ $(-1,5,3), \mathcal{X}(0)=(3,-6,2), \mathcal{X}(0)=(-2,1,4), \mathcal{X}(0)=$ $(-2.1,3.7,-1)$. Figure 3 and 4 shows the corresponding trajectories when $r=-10$. It's also interesting to notice the role of the gain $\kappa$. In particular for high-gain, the transient is faster but the error of the output tracking is higher in the transient. On the contrary, for low-gain value the transient is longer but the error of the output during is lower.

\section{Conclusions}

In this paper we have studied the output set-point tracking problem for nonlinear systems by following the classical linear output regulation approach, see [15]. First, we extended the system with an integral action processing the regulated output; then we designed a stabilizing feedback law for the extended system by supposing the reference to be zero; finally, we studied sufficient conditions guaranteeing that when the reference is non-zero, the tracking problem is solved. In doing so, we presented a number of new results. First, we addressed the incremental stabilization problem via forwarding by providing two different sets of sufficient conditions and constructive design in order to obtain (non-uniform) incremental exponential stability and global incremental exponential stability. Then, we studied the effect of large tracking references. In particular, we showed that, because of the contractivity properties of the system, if the trajectories of the closed-loop system are bounded in forward time, then necessarily the tracking objective is achieved. Finally, sufficient conditions to solve the global output tracking problem are given.

Future works may include a robust analysis with respect to model perturbations in the same spirit of [6], [10], the design of an output feedback law [6], and the extension of the proposed technique to the case of periodic references, as done in [7], [8] or [28].

${ }^{2}$ See [18, Chapter 4.1].
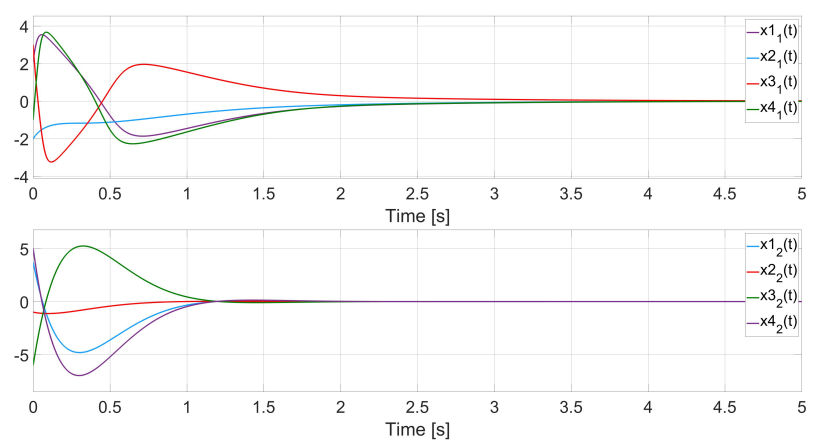

Fig. 1. $x$-dynamic in stabilization

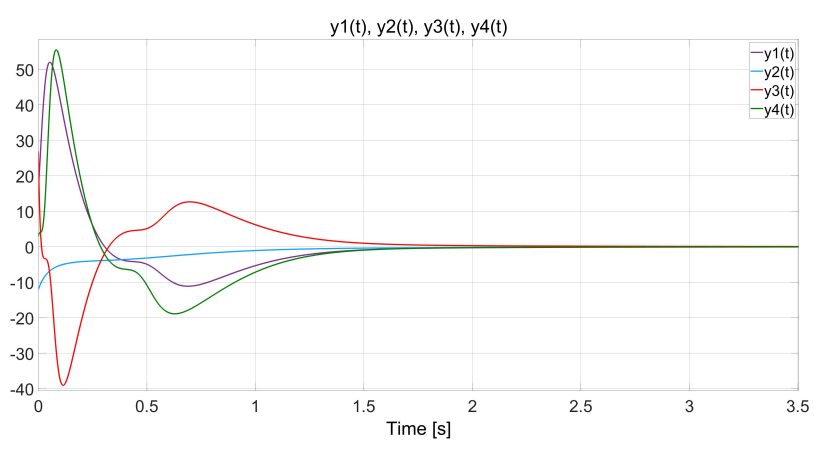

Fig. 2. $y$-dynamic in stabilization

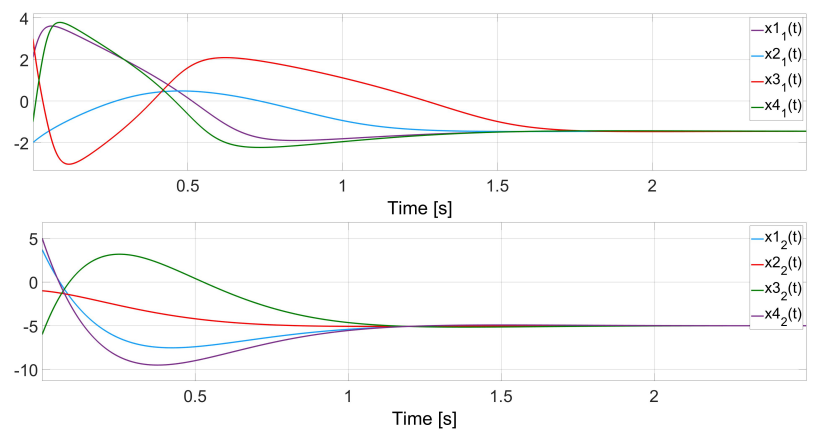

Fig. 3. $x$-dynamic in tracking

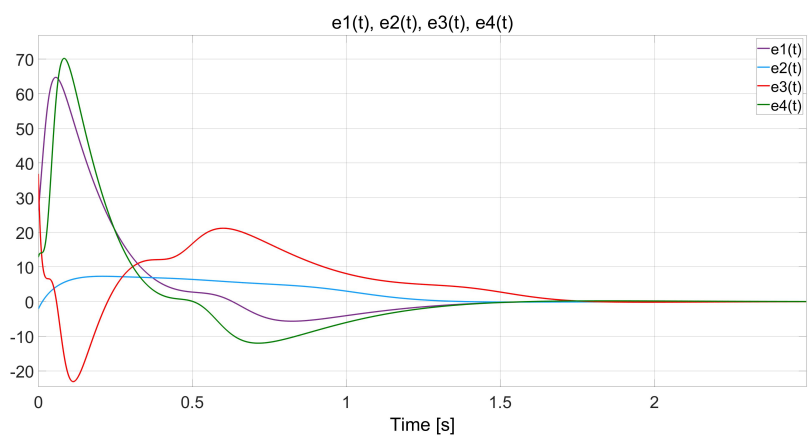

Fig. 4. Tracking error dynamic 


\section{APPENDIX}

\section{A. Proof of Lemma 1}

Let $\tau$ be such that $k \exp (-\lambda \tau)=\gamma<1$ and define $\mathcal{T}_{\tau}$ as the mapping that associates to any initial condition $\mathcal{X}_{0}$ of system of (6), its corresponding solution $\mathcal{X}\left(\mathcal{X}_{0}, \tau\right)$ at time $\tau$, that is $\mathcal{T}_{\tau}(\mathcal{X}):=\mathcal{X}\left(\mathcal{X}_{0}, \tau\right)$. Since $\mathcal{C}$ is forward invariant, the function so defined maps points in $\mathcal{C}$ into $\mathcal{C}$. Furthermore, by using the inequality (8) guaranteed by the IES property of system (6) on $\mathcal{C}$, for any two given points $\mathcal{X}_{1}, \mathcal{X}_{2} \in \mathcal{C}$, we have

$$
\left|\mathcal{T}_{\tau}\left(\mathcal{X}_{1}\right)-\mathcal{T}_{\tau}\left(\mathcal{X}_{2}\right)\right| \leq \rho\left|\mathcal{X}_{1}-\mathcal{X}_{2}\right|
$$

with $\rho<1$. Recall that the Euclidean space endowed with standard Euclidean norm is a complete metric. Hence, the map $\mathcal{T}_{\tau}$ is a contraction and by Banach fixed point theorem (see, e.g., [1]), there exists a unique fixed point $x^{\star}$ in $\mathcal{C}$ such that $\mathcal{T}_{\tau}\left(x^{\star}\right)=x^{\star}$. Hence, for any $\mathcal{X}$ in $\mathcal{C}$, inequality (8), implies,

$$
\begin{aligned}
\left|\mathcal{X}(\mathcal{X}, t)-\mathcal{X}^{\star}\right| & =\left|\mathcal{X}(\mathcal{X}, t)-\mathcal{X}\left(\mathcal{X}^{\star}, t\right)\right| \\
& \leq k \exp (-\lambda t)\left|\mathcal{X}-\mathcal{X}^{\star}\right| .
\end{aligned}
$$

Hence, $\mathcal{X}^{\star}$ is attracts all trajectories initiated from $\mathcal{C}$.

An equivalent proof of this lemma, covering also the case of periodic equilibrium, has been developed independently in $[16$, Section IV-B].

\section{B. Proof of Theorem 3}

In the new coordinates $\overline{\mathcal{X}}=(x, z)$, where $z=\eta-M(x)$ with $M$ given by Lemma 2, (4) becomes

$$
\dot{\overline{\mathcal{X}}}=\bar{\varphi}(\overline{\mathcal{X}})=\left[\begin{array}{c}
f(x)+\kappa g(x) L_{g} M(x)^{-1} \beta(z) \\
\kappa \beta(z)
\end{array}\right] .
$$

The Jacobian of $\bar{\varphi}$ is given by

$$
\frac{\partial \bar{\varphi}}{\partial \overline{\mathcal{X}}}(\overline{\mathcal{X}})=\left[\begin{array}{cc}
J_{11} & J_{12} \\
0 & J_{22}
\end{array}\right]
$$

where

$$
\begin{aligned}
J_{11} & =\frac{\partial f}{\partial x}(x)-\kappa g(x) \frac{\partial L_{g} M}{\partial x}(x) \frac{\beta(z)}{L_{g} M(x)^{2}}, \\
J_{12} & =\kappa g(x) \frac{\beta^{\prime}(z)}{L_{g} M(x)} \\
J_{22} & =-\kappa \beta^{\prime}(z) .
\end{aligned}
$$

Let $\overline{\mathcal{P}}(x)=\overline{\mathcal{P}}(x)^{\top}>0$ be defined as

$$
\overline{\mathcal{P}}(x)=\left[\begin{array}{cc}
P(x) & 0 \\
0 & \mathfrak{b}
\end{array}\right],
$$

for some $\mathfrak{b} \in \mathbb{R}_{>0}$ to be defined later. Let also $\mathcal{R}$ be the matrix defined as

$$
\mathcal{R}(\overline{\mathcal{X}})=L_{\bar{\varphi}} \overline{\mathcal{P}}(\overline{\mathcal{X}})+\left[\begin{array}{cc}
\frac{q}{2} I_{n} & 0 \\
0 & \kappa \beta^{\prime}(z)
\end{array}\right] .
$$

We show that $\mathcal{R}$ takes only negative definite values provided $\kappa$ is selected sufficiently small. To do this, note that

$$
\mathcal{R}(\overline{\mathcal{X}})=\left[\begin{array}{ll}
L_{11} & L_{12} \\
L_{12}^{\top} & L_{22}
\end{array}\right]
$$

where, employing Assumption 3,

$$
\begin{aligned}
L_{11}= & L_{f} P(x)+\kappa\left(P(x) g(x) \frac{\partial L_{g} M}{\partial x}(x) \frac{\beta(z)}{L_{g} M(x)^{2}}\right)^{\top} \\
& \quad+\kappa\left(P(x) g(x) \frac{\partial L_{g} M}{\partial x}(x) \frac{\beta(z)}{L_{g} M(x)^{2}}\right)+\frac{q}{2} I_{n}, \\
L_{12}= & \mathfrak{b} \kappa P(x) g(x) \frac{\beta^{\prime}(z)}{L_{g} M(x)}, \\
L_{22}= & -\mathfrak{b} \kappa \beta^{\prime}(z) .
\end{aligned}
$$

Since, $L_{22}<0, \mathcal{R}$ is negative definite provided its Schur complement $L_{11}-\frac{L_{12} L_{12}^{\top}}{L_{22}}$ is so. Note that, with Assumption 1, Assumption 2, inequalities (17) and the bound on $\beta$ selected in (18), we have

$$
L_{11} \leq\left(2 \kappa \frac{\bar{p} \bar{g}}{m^{2}}-\frac{q}{2}\right) I_{n} .
$$

Moreover, with Assumption 2 and the fact that $\beta^{\prime}(z)>0$,

$$
\left|L_{12}\right| \leq \mathfrak{b} \kappa \frac{\bar{p} \bar{g}}{m} \beta^{\prime}(z) .
$$

It implies

$$
L_{11}-\frac{L_{12} L_{12}^{\top}}{L_{22}} \leq\left(2 \kappa \frac{\bar{p} \bar{g}}{m^{2}}-\frac{q}{2}\right) I_{n}+\mathfrak{b} \kappa \frac{\bar{p}^{2} \bar{g}^{2}}{m^{2}} \beta^{\prime}(z),
$$

namely, with the bound on $\beta^{\prime}$,

$$
L_{11}-\frac{L_{12} L_{12}^{\top}}{L_{22}} \leq\left(\kappa \frac{\bar{p} \bar{g}}{m^{2}}(2+\mathfrak{b} \bar{p} \bar{g})-\frac{q}{2}\right) I_{n} .
$$

Selecting $\mathfrak{b}=1$ and $\kappa^{*}$ as

$$
\kappa^{*}=\frac{q m^{2}}{2 \bar{p} \bar{g}(2+\bar{p} \bar{g})},
$$

it yields $\mathcal{R}$ negative definite and consequently, with (26),

$$
L_{\bar{\varphi}} \overline{\mathcal{P}}(\overline{\mathcal{X}}) \leq-\min \left\{\frac{q}{2}, \kappa \beta^{\prime}(|z|)\right\} I_{n+1} .
$$

Going back into the original coordinates $\mathcal{X}=(x, \eta)$, inequality (9) holds with

$$
\begin{aligned}
\mathcal{P}(\mathcal{X}) & =\left[\begin{array}{cc}
I_{n} & -\frac{\partial M}{\partial x}(x)^{\top} \\
0 & 1
\end{array}\right]\left[\begin{array}{cc}
P(x) & 0 \\
0 & \mathfrak{b}
\end{array}\right]\left[\begin{array}{cc}
I_{n} & 0 \\
-\frac{\partial M}{\partial x}(x) & 1
\end{array}\right] \\
& =\left[\begin{array}{cc}
P(x)+\mathfrak{b} \frac{\partial M}{\partial x}(x)^{\top} \frac{\partial M}{\partial x}(x) & -\mathfrak{b} \frac{\partial M}{\partial x} \\
-\mathfrak{b} \frac{\partial M}{\partial x}(x) & \mathfrak{b}
\end{array}\right],
\end{aligned}
$$

$\overline{\mathfrak{p}}=\max \left\{\bar{p}+\mathfrak{b} k_{0}^{2}, \mathfrak{b}\right\}, \mathfrak{p}=\min \left\{\underline{p}+\mathfrak{b} k_{0}^{2}, \mathfrak{b}\right\}$. Then, inequality (19) holds with such $\overline{\mathcal{P}}$ and the function $\mu$ defined as

$$
\mu(\eta, x)=\frac{1}{\left(1+k_{0}\right)^{2}} \min \left\{\frac{q}{2}, \kappa \beta^{\prime}(|\eta-M(x)|)\right\} .
$$

For the last part of the theorem, since $\mathcal{P}$ doesn't depend on $\eta$, it yields that $\mathfrak{d}_{\Gamma} \mathcal{P}(\mathcal{X})=0$. Consequently, $L_{\Gamma} \mathcal{P}(\mathcal{X})=0$. 


\section{Proof of Theorem 4}

The proof goes along the same lines of the one of Theorem 3. Indeed, in this case,

$$
L_{11}=L_{f} P(x)+\frac{q}{2} I_{n} \leq-\frac{q}{2} I_{n}
$$

regardless the function $\beta$ and $\kappa$, i.e. the control law has infinite gain margin [31, Definition 2.8]. By taking $\beta(s)=s$, inequality (29) modifies as

$$
L_{11}-\frac{L_{12} L_{12}^{\top}}{L_{22}} \leq-\frac{q}{2} I_{n}+\mathfrak{b} \kappa \frac{\bar{p}^{2} \bar{g}^{2}}{m^{2}} .
$$

For any given $\kappa$, select

$$
\mathfrak{b}=\frac{q m^{2}}{4 \kappa \bar{p}^{2} \bar{g}^{2}}
$$

This implies that equation (30) becomes

$$
L_{\bar{\varphi}} \overline{\mathcal{P}}(\overline{\mathcal{X}}) \leq-\min \left\{\frac{q}{2}, \kappa\right\} I_{n+1},
$$

and, consequently, (32) becomes

$$
\mu(\eta, x)=\mu=\frac{1}{\left(1+k_{0}\right)^{2}} \min \left\{\frac{q}{2}, \kappa\right\} .
$$

Hence, the statement of the theorem follows with $\overline{\mathfrak{p}}, \mathfrak{p}$ as in the proof of Theorem 3 and $\mathfrak{q}=\mu$ defined above. Finally, with the same arguments of the proof of Theorem 3, we have $L_{\Gamma} \mathcal{P}(\mathcal{X})=0$.

\section{REFERENCES}

[1] Saleh Almezel, Qamrul Hasan Ansari, and Mohamed Amine Khamsi. Topics in fixed point theory, volume 5. Springer, 2014.

[2] V. Andrieu, B. Jayawardhana, and L Praly. Transverse exponential stability and applications. IEEE Transactions on Automatic Control, 61(11):3396-3411, 2016.

[3] V. Andrieu, B. Jayawardhana, and S. Tarbouriech. Some results on exponential synchronization of nonlinear systems. IEEE Transactions on Automatic Control, 63(4):1213-1219, 2018.

[4] V. Andrieu and S. Tarbouriech. LMI conditions for contraction and synchronization. IFAC-PapersOnLine, 52(16):616-621, 2019.

[5] D. Angeli. A lyapunov approach to incremental stability properties. IEEE Transactions on Automatic Control, 47(3):410-421, 2002.

[6] D. Astolfi and L. Praly. Integral action in output feedback for multiinput multi-output nonlinear systems. IEEE Transactions on Automatic Control, 62(4):1559-1574, 2017.

[7] D. Astolfi, L. Praly, and L. Marconi. Approximate regulation for nonlinear systems in presence of periodic disturbances. In 54th IEEE Conference on Decision and Control, pages 7665-7670. IEEE, 2015.

[8] D. Astolfi, L. Praly, and L. Marconi. Francis-wonham nonlinear viewpoint in output regulation of minimum phase systems. IFACPapersOnLine, 52(16):532-537, 2019.

[9] S. Behtash. Robust output tracking for non-linear systems. International Journal of Control, 51(6):1381-1407, 1990.

[10] M. Bin, D. Astolfi, L. Marconi, and L. Praly. About robustness of internal model-based control for linear and nonlinear systems. In 57th IEEE Conference on Decision and Control, pages 5397-5402, 2018.

[11] M. Bin and L. Marconi. Output regulation by postprocessing internal models for a class of multivariable nonlinear systems. International Journal of Robust and Nonlinear Control, 30(3):1115-1140, 2020.

[12] S. Carroll. Spacetime and geometry: An introduction to general relativity. 2004.

[13] L. DAlto and M. Corless. Incremental quadratic stability. Numerical Algebra, Control and Optimization, 3(1):175-201, 2013.

[14] F. Forni and R. Sepulchre. A differential lyapunov framework for contraction analysis. IEEE Transactions on Automatic Control, 59(3):614-628, 2013.

[15] B.A. Francis and W.M. Wonham. The internal model principle of control theory. Automatica, 12(5):457-465, 1976.
[16] W.P.M.H. Heemels, M.K. Camlibel, and M.F. Heertjes. Oblique projected dynamical systems and incremental stability under state constraints. IEEE Control Systems Letters, 2020.

[17] X. Huang, H.K. Khalil, and Y. Song. Regulation of nonminimumphase nonlinear systems using slow integrators and high-gain feedback. IEEE Transactions on Automatic Control, 64(2):640-653, 2018.

[18] A. Isidori. Nonlinear Control Systems. Springer, 1995.

[19] M. Jankovic, R. Sepulchre, and P.V. Kokotovic. Constructive lyapunov stabilization of nonlinear cascade systems. IEEE transactions on automatic control, 41(12):1723-1735, 1996.

[20] ZP. Jiang and I. Marcels. Robust nonlinear integral control. IEEE Transactions on Automatic Control, 46(8):1336-1342, 2001.

[21] G. Kaliora and A. Astolfi. Nonlinear control of feedforward systems with bounded signals. IEEE Transactions on Automatic Control, 49(11):1975-1990, 2004.

[22] H.K. Khalil. Universal integral controllers for minimum-phase nonlinear systems. IEEE Transactions on automatic control, 45(3):490-494, 2000

[23] H.K. Khalil. Nonlinear systems, volume 3. Prentice hall, 2002.

[24] DC. Lewis. Metric properties of differential equations. American Journal of Mathematics, 71(2):294-312, 1949.

[25] W. Lohmiller and JJ. E. Slotine. On contraction analysis for non-linear systems. Automatica, 34(6):683-696, 1998.

[26] R. Marino and P. Tomei. Output regulation for unknown stable linear systems. IEEE Transactions on Automatic Control, 60(8):2213-2218, 2014.

[27] F. Mazenc and L. Praly. Adding integrations, saturated controls, and stabilization for feedforward systems. IEEE Transactions on Automatic Control, 41(11):1559-1578, 1996.

[28] A. Pavlov and L. Marconi. Incremental passivity and output regulation. Systems \& Control Letters, 57(5):400-409, 2008.

[29] A. Pavlov, N. van de Wouw, and H. Nijmeijer. Uniform output regulation of nonlinear systems: a convergent dynamics approach. Springer Science \& Business Media, 2006.

[30] F. Poulain and L. Praly. Robust asymptotic stabilization of nonlinear systems by state feedback. IFAC Proceedings Volumes, 43(14):653$658,2010$.

[31] R. Sanfelice and L. Praly. Convergence of nonlinear observers on $\mathbb{R}^{n}$ with a riemannian metric (part i). IEEE Transactions on Automatic Control, 57(7):1709-1722, 2011.

[32] BB. Sharma and IN. Kar. Contraction theory based adaptive synchronization of chaotic systems. Chaos, Solitons \& Fractals, 41(5):24372447, 2009.

[33] S. Waitman, L. Bako, P. Massioni, G. Scorletti, and V. Fromion Incremental stability of lure systems through piecewise-affine approximations. IFAC-PapersOnLine, 50(1):1673-1679, 2017.

[34] L. Wang, L. Marconi, C. Wen, and H. Su. Pre-processing nonlinear output regulation with non-vanishing measurements. Automatica, 111:108616, 2020.

[35] V.A. Yakubovich. Matrix inequalities method in stability theory for nonlinear control systems: I. absolute stability of forced vibrations. Automation and remote control, 7(905-917):202, 1964.

[36] M. Zamani and P. Tabuada. Backstepping design for incremental stability. IEEE Transactions on Automatic Control, 56(9):2184-2189, 2011.

[37] M. Zamani, N. van de Wouw, and R. Majumdar. Backstepping controller synthesis and characterizations of incremental stability. Systems \& Control Letters, 62(10):949-962, 2013. 